\title{
Exploring COVID-19 Therapies: An Extraordinary Global Challenge
}

\author{
Srikant Mohta ${ }^{1}$ Ashish Agarwal ${ }^{1}$ Govind K. Makharia ${ }^{1, \odot}$ \\ ${ }^{1}$ Department of Gastroenterology and Human Nutrition, \\ All India Institute of Medical Sciences, New Delhi, India \\ Address for correspondence Govind K. Makharia, MD, DM, DNB, \\ MNAMS, Department of Gastroenterology and Human Nutrition, \\ All India Institute of Medical Sciences, Ansari Nagar, New Delhi 110029, \\ India (e-mail: govindmakharia@gmail.com, govindmakharia@aiims.edu).
}

| Digest Endosc 2020;11:73-75

We are in the midst of a global pandemic of novel SARS CoV-2 viral infection which have affected more than 2.6 million people in 180 countries and has already claimed nearly 180,000 lives. ${ }^{1}$ The magnitude of devastation and sense of fear and uncertainty caused by the pandemic is unprecedented. It is extremely motivating to see the efforts by everyone to win over this global crisis; such as governments trying to flatten the curve and trading off between loss of human lives and big jolt to their economies; health care workers providing care to their patients and adapting to their new attire (personal protective equipment [PPE]); scientists, both fundamental and clinical, and pharmaceutical companies exploring the disease biology and potential drug targets and vaccines; disease epidemiologists defining the appropriate strategies for the containment of the virus; regulatory bodies clearing clinical trials and scientific studies at a neck-breaking speed; police and authorities enforcing social distancing; scientific societies imparting knowledge about this disease and creating specific guidelines; the publishing houses disseminating relevant knowledge by very fast track online publication(s); and the countrymen cheering the COVID warriors in innovative ways and staying at home. This pandemic is also marked by exposure of weak side of humanity such as game of blames, rumors, stockpiling and hoarding, and assaults on health care professionals and policemen. While this pandemic brought great challenges, it however also brought the whole world together.

Health care professionals worldwide are trying to beat this pandemic by exploring novel ideas and innovations to explore and establish appropriate management strategies for COVID-19. There is flurry of new knowledge almost every day and it is hard to keep a track of all that is new, especially in the therapeutics of COVID-19. That being said, the review by Kant Kumar et al published in this journal is a welcome manuscript which adequately summarizes the available therapeutic options as of now and briefly touches upon the status of vaccine development. ${ }^{2}$

It is very reassuring that majority of patients with COVID-19 disease have a mild course and only approximately 10 to $15 \%$ of them require substantial health care facility and specific treatment. ${ }^{3}$ Therefore, the mainstay of treatment of COVID-19 is supportive for all the stages of the disease and use of lung protective ventilation, Surviving Sepsis Guidelines for COVID, and investigational agents only in a few selected patients. ${ }^{4,5}$

Talking about the extraordinary efforts to explore appropriate treatment of SARS-CoV-2 infection, 745 studies are registered on ClinicalTrials.gov as of April 22, 2020. Of them, 342 are clinical trials. ${ }^{6}$

The investigational drugs which are being explored to target different steps of SARS-CoV-2 lifecycle in humans including inhibition of entry of virus into the target cell by inhibition of angiotensin-converting enzyme- 2 receptor and transmembrane protease serine-2 (TMPRSS2) by Olumiant (baricitinib) $)^{7}$ and camostat mesylate, ${ }^{8}$ respectively; inhibition of the viral entry by inhibition of the membrane fusion and endocytosis by chloroquine or hydroxychloroquine ${ }^{9}$ and umifenovir (arbidol) $)^{10}$; inhibition of viral replication by protease inhibitors such as lopinavir ${ }^{11}$ and darunavir; and blockade of viral ribonucleic acid (RNA) synthesis by remdesivir, ${ }^{12}$ ribavirin, ${ }^{13}$ and favipravir. ${ }^{14}$ The other class of drugs control the hyperinflammatory phase such as corticosteroids, immunomodulators, and anti-cytokines such as interleukin (IL)-6 inhibitor tocilizumab. ${ }^{15}$

Hydroxychloroquine has been the most hyped drug in the recent times and this drug has been approved by the U.S. Food and Drug Administration, European Medical Association, and Indian Council for Medical Research for its use in the treatment of COVID-19 patients as a desperate measure even in the absence of strong supportive evidence of its efficacy. Hydroxychloroquine has also been approved for use as a prophylaxis in asymptomatic health care workers involved in the care of suspected or confirmed patients with COVID-19 and asymptomatic household contacts of laboratory-confirmed COVID-19 patients. ${ }^{16}$ One must remember that use of these drugs is known to prolong QT interval and fatal arrhythmia. Nevertheless, as of now at least 10 clinical trials are underway and the results of at least some of them should be available over a few weeks to
DOI https://doi.org/

10.1055/s-0040-1712598

ISSN 0976-5042.
License terms

()(1) $\Theta \circledast$ 
months which will enable us to make more evidence-based decisions.

Antiviral drugs have been considered to be one of the front-runner drugs; however, lopinavir/ritonavir combination in comparison to standard of care was neither associated with a clinical improvement, nor mortality at 28 days in a randomized controlled trial including 199 patients with COVID-19. ${ }^{11}$ Remdesivir is another major drug which has already been approved for Ebola virus infection. While remdesivir has shown its efficacy in a small nonrandomized study, ${ }^{12}$ provisional report of a phase 3 clinical trial, currently being done in Chicago, United States, has also confirmed its efficacy in moderate and severe COVID-19 patients. ${ }^{17}$ Based on these early clinical results, remdesivir has been approved for its use on a compassionate ground in appropriate patients awaiting confirmation of its efficacy at the completion of the ongoing study. Other antiretroviral drugs such as darunavir/cobicistat are also being evaluated for its efficacy in COVID-19 patients. ${ }^{15}$

Although in the review by Kant Kumar et al, they have comprehensively covered most of the main pipeline drugs, a few more, we believe, are worthy of a mention here. ${ }^{2}$ As we said earlier, in this unprecedented time, authors are relaying on the results of their studies through prepublication portal till the paper is peer-reviewed. One of such drug is favipravir which is a RNA polymerase inhibitor drug and it has shown its efficacy against influenza virus and Ebola virus infection. In a multicenter study including 240 patients with moderate and severe COVID-19, favipravir has shown good results in comparison to another drug umifenovir (arbidol). ${ }^{14}$

The SARS-CoV2 viral infection may have three phases, including early viral phase, pulmonary phase, and it may pass on the to the most fatal hyperinflammation phase in a small subset of patients. ${ }^{18}$ The hyperinflammation phase is critical and it is characterized by cytokine storm and thus attracting therapies such as corticosteroids, immunomodulators, and anti-cytokines therapy. The hyperinflammatory phase is uniquely characterized by high level of IL-6 and hence early result of tocilizumab (400 mg single dose), an IL-6 receptor antagonist, in 21 patients of an ongoing clinical trial in China has demonstrated an improvement in clinical condition in $75 \%$ and radiological resolution in $90.5 \%$ of patients. ${ }^{19,20}$

Another adjunctive therapy showing promise in COVID-19 is immunoglobulin administration from convalescent plasma of recovered individuals thus providing passive immunization. ${ }^{15}$ It has been successfully used earlier in the treatment of SARS, MERS, and 2009 H1N1 pandemic with good efficacy and safety. A meta-analysis of 32 studies reporting outcome of use of convalescent plasma in patients with influenza and SARS has shown a consistent reduction in mortality, especially if administered early after symptom onset. ${ }^{11}$ Convalescent plasma is one of the most promising options as a rescue treatment for severe cases of COVID-19 and it is being explored at least in 17 ongoing trials worldwide, including some Indian centers. ${ }^{6}$

Prevention of infection by augmenting immunity against SARS-CoV-2 virus using vaccine is the ultimate long-term goal. As highlighted by Kant Kumar et al, several vaccine are being explored with some starting early clinical trials, the availability of an effective vaccine is not close and it may take 9 to 12 months for mass production and mass use. ${ }^{2}$ We will have to wait for results of ongoing trials before specific treatment and preventive strategies are established as a standard of care, and till then, prevention of the spread of the disease remains at the center stage.

In conclusion, an extraordinary effort is being made by the governments, scientists, and health care professionals to unravel a magic drug or a target to protect human kind from this viral pandemic. While we wait for the brighter side, we in the meanwhile should rise to our best in discharging our duties as responsible health care professionals. While we are learning and adapting to the new ways of treating our patients in the COVID and post-COVID era, we surely have learnt three buzz words, namely social distancing, PPE, and flattening of the curve.

Humanity at its best and cheers!

\section{Authors' Contributions}

S.M.-Drafting of the manuscript and review.

A.A.-Drafting of the manuscript and review.

G.M.-Critical revision of the manuscript for important intellectual content.

Each of the authors has approved the final draft of the manuscript submitted.

\section{Financial Support}

None.

\section{Conflict of Interest}

There is no conflict of interest to declare.

\section{References}

1 Coronavirus Update (Live). 2,660,763 Cases and 185,502 Deaths from COVID-19 Virus Pandemic - Worldometer. Available at: https://www.worldometers.info/coronavirus/. Accessed April 23, 2020

2 Jha A, Kant Kumar R, Goenka MK, Dayal VM. Emerging treatment and prevention strategies against COVID-19:a brief update. J Dig Endosc 2020;11;69-72

3 Verity R, Okell LC, Dorigatti I, et al. Estimates of the severity of coronavirus disease 2019: a model-based analysis. Lancet Infect Dis 2020;S1473-3099(20)30243-710.1016/S14733099(20)30243-7

4 Alhazzani W, Møller MH, Arabi YM, et al. Surviving Sepsis Campaign: guidelines on the management of critically ill adults with Coronavirus Disease 2019 (COVID-19) Intensive Care Med 2020;10.1007/s00134-020-06022-5

5 Phua J, Weng L, Ling L, et al; Asian Critical Care Clinical Trials Group. Intensive care management of coronavirus disease 2019 (COVID-19): challenges and recommendations. Lancet Respir Med 2020;:S2213-2600(20)30161-210.1016/S22132600(20)30161-2

6 Home - ClinicalTrials.gov. Available at: https://clinicaltrials. gov/. Accessed April 22, 2020

7 Richardson P, Griffin I, Tucker C, et al. Baricitinib as potential treatment for 2019-nCoV acute respiratory disease. Lancet 2020;395(10223) :e30-e31

8 Shirato K, Kawase M, Matsuyama S. Middle East respiratory syndrome coronavirus infection mediated by the transmembrane serine protease TMPRSS2. J Virol 2013;87(23):12552-12561 
9 Singh AK, Singh A, Shaikh A, Singh R, Misra A. Chloroquine and hydroxychloroquine in the treatment of COVID-19 with or without diabetes: a systematic search and a narrative review with a special reference to India and other developing countries. Diabetes Metab Syndr 2020;14(3):241-246

10 Teissier E, Zandomeneghi G, Loquet A, et al. Mechanism of inhibition of enveloped virus membrane fusion by the antiviral drug arbidol. PLoS One 2011;6(1):e15874

11 Cao B, Wang Y, Wen D, et al. A trial of lopinavir-ritonavir in adults hospitalized with severe COVID-19. N Engl J Med 2020;10.1056/NEJMoa2001282

12 Grein J, Ohmagari N, Shin D, et al. Compassionate use of remdesivir for patients with severe COVID-19. N Engl J Med 2020;0(0)10.1056/NEJMoa2007016

13 Khalili JS, Zhu H, Mak NSA, Yan Y, Zhu Y. Novel coronavirus treatment with ribavirin: Groundwork for an evaluation concerning COVID-19. J Med Virol 2020;10.1002/jmv.25798

14 Chen C, Zhang Y, Huang J, et al. Favipiravir versus arbidol for COVID-19: a randomized clinical trial. medRxiv 2020;2020.0.1 7.2003743210.1101/2020.03.17.20037432

15 Sanders JM, Monogue ML, Jodlowski TZ, Cutrell JB. Pharmacologic treatments for coronavirus disease 2019 (COVID-19): a review. JAMA 2020;10.1001/jama.2020.6019
16 Advisory on the use of Hydroxychloroquinasprophylaxis for SARSCoV2 infection.pdf. Available at: https://www.mohfw.gov. in/pdf/Advisory on the use of Hydroxychloroquinasprophylaxis for SARS CoV2 infection. pdf. Accessed April 23, 2020

17 Gilead's remdesivir yields promising data in COVID-19 trial. Clinical Trials Arena. Published April 17, 2020. Available at: https://www.clinicaltrialsarena.com/news/gilead-remdesivir-covid-trial-data/. Accessed April 23, 2020

18 Mason RJ. Pathogenesis of COVID-19 from a cell biology perspective. Eur Respir J 2020;55(4):2000607

19 Xu X, Han M, Li T, et al. Effective treatment of severe COVID-19 patients with tocilizumab. chinaXiv 2020

20 Mair-Jenkins J, Saavedra-Campos M, Baillie JK, et al; Convalescent Plasma Study Group. The effectiveness of convalescent plasma and hyperimmune immunoglobulin for the treatment of severe acute respiratory infections of viral etiology: a systematic review and exploratory meta-analysis. J Infect Dis 2015;211(1):80-90 